\title{
Inflammation, genetic background and longevity
}

\author{
Giuseppina Candore • Calogero Caruso • \\ Giuseppina Colonna-Romano
}

Received: 5 February 2010/Accepted: 2 June 2010/Published online: 13 June 2010

(C) Springer Science+Business Media B.V. 2010

\begin{abstract}
Ageing is an inexorable intrinsic process that affects all cells, tissues, organs and individuals. Due to a diminished homeostasis and increased organism frailty, ageing causes a reduction of the response to environmental stimuli and, in general, is associated to an increased predisposition to illness and death. Actually, it is characterized by a state of reduced ability to maintain health and general homeodynamics of the organism. A large part of the ageing phenotype is explained by an imbalance between inflammatory and anti-inflammatory networks, which results in the low grade chronic pro-inflammatory status of ageing, "inflamm-ageing". It is strictly linked to immunosenescence, and on the whole they are the major contributory factors to the increased frequency of morbidity and mortality among elderly. Inflammageing is compatible with longevity; even if centenarians have an increased level of inflammatory mediators in comparison to old subjects and they are very frail, they also have high level of anti-inflammatory cytokines together with protective genotypes. Actually, data on case control studies performed in Italian centenarians suggest that a pro-inflammatory genotype is unfavourable to reach extreme longevity in good
\end{abstract}

G. Candore · C. Caruso $(\bowtie) \cdot$ G. Colonna-Romano Immunopathology Laboratory, Department of Pathobiology and Medical and Forensic Biotechnologies, University of Palermo, Corso Tukory 211, 90134 Palermo, Italy

e-mail: immunopatologia@unipa.it; marcoc@unipa.it health and likely favours the onset of age-related diseases such as cardiovascular diseases and Alzheimer's disease, the leading causes of mortality and disability in the elderly. However, many associations between gene variants and longevity have been found only in Italian population. This should not be unexpected, since ageing and longevity are complex traits resulting not only and not exclusively from genetics, but rather from the interactions between genetics, environment and chance.

Keywords Immunogenetics · Immunosenescence · Inflammation · Longevity

\section{Ageing}

Ageing is an inexorable intrinsic process that affects all cells, tissues, organs and individuals. Due to a diminished homeostasis and increased organism frailty, ageing causes a reduction of the response to environmental stimuli and, in general, is associated to an increased predisposition to illness and death. Actually, it is characterized by a state of reduced ability to maintain health and general homeodynamics of the organism (Troen 2003; Rattan 2008).

Nowadays, many countries have rising ageing populations and are facing an increased prevalence of age-related diseases and increasing healthcare costs. During the twentieth century, life expectancy at birth 
rose by a remarkable 30 years in developed countries, initially because of reductions in infant, child, and maternal mortality and then because of declining mortality in middle and old age. In 1900, about 40\% of babies born in countries for which reliable data existed were expected to live beyond age 65 . Today in these same countries more than $88 \%$ of all newborns will live past age 65 and at least $44 \%$ will live beyond age 85 . However, the rapid rise in elderly people is accompanied by an increase in the number of people with chronic age-related diseases since elderly are less resistant to both environmental and pathological injury; in addition, they are less resistant to infection (Olshansky and Ault 1986; Oeppen and Vaupel 2002).

Why we age is a question answered by evolutionary biologists in terms of allocation of resources, and by gerontologists in terms of damage and repair. However, the ageing phenotype is a complex interaction of stochastic, environmental, genetic and epigenetic variables. These variables do not create the ageing phenotype but generate the lack of molecular fidelity and therefore as the random accumulation of damage in the human organism's cells, tissues, or whole organism during life increases, the probability of disease and death also augments in proportion (Troen 2003; Rattan 2008).

As discussed in the present paper, a large part of the ageing phenotype is explained by an imbalance between inflammatory and anti-inflammatory networks, which results in the low grade chronic proinflammatory status of ageing (Licastro et al. 2005; Vasto et al. 2007).

\section{Inflammation and ageing}

Inflammation is defined as a localized response with systemic consequences, elicited by trauma or infection, which helps to destroy, reduce or sequester both the harmful agent and the wounded tissue. In response to stable low-grade irritation, inflammation becomes a chronic condition that continuously damages the surrounding tissues, and it occurs when the tissues are unable to overcome the effects of the harmful agent (Licastro et al. 2005). However, the inflammation process is not per se a negative phenomenon: it is the response of the immune system to pathogenic viruses or bacteria. Throughout evolution, humans have been set to live approximately 40 or 50 years; however, today, the immune system has to be active longer than in the past centuries. This prolonged period of activation may lead to chronic inflammation, which inexorably damages several/all organs, and this phenotype is linked to both ageing and chronic diseases (Vasto et al. 2007; Candore et al. 2009, 2010a).

Thus, human ageing can be explained at least in part by the concept of "inflamm-ageing", i.e. a combination of inflammation and ageing (Franceschi et al. 2007). Inflamm-ageing posits that ageing either physiologically or pathologically can be driven by the pro-inflammatory cytokines and other inflammatory mediators produced by the innate immune system. Animals must maintain homeostasis as they age despite incessant attack from both intrinsic and extrinsic stimuli/antigens. These potentially harmful pro-inflammatory signals at a later stage of life may act antagonistically to the beneficial role they had in an earlier stage of life. Accordingly, the concept of inflamm-ageing is based on an antagonistic pleiotropy theory programmed during evolution (Goto 2008; Cevenini et al. 2010).

In fact, ageing is accompanied by chronic lowgrade inflammation state, showed by a 2 to 4 -fold increase in serum levels of inflammatory mediators which act as predictors of mortality independent on pre-existing morbidity. Low grade inflammation has emerged as critical in the pathogenesis of several agerelated chronic diseases as Alzheimer's disease (AD), cardiovascular disease (CVD), type 2 diabetes, sarcopenia, frailty and functional disability. These diseases appear to be correlated, leading to the concept of age-related disease, which may be linked by the process of inflammation (Candore et al. 2009; Vasto et al. 2010). So, genes and epigenetic factors involved in the regulatory pattern of inflammation should play a role in human ageing (Vasto et al. 2007; Cevenini et al. 2010). Furthermore high serological levels of pro-inflammatory mediators like cytokines and $\mathrm{C}$ reactive protein (CRP) seem to be the marker of unsuccessful ageing (Vasto et al. 2010). In a recent study blood levels of CRP, interleukin (IL)-6, IL-1 receptor antagonist (IL-1ra) and their combinations, as predictors of mortality during 4 years, have been investigated in nonagenarians. Plasma levels of IL1ra, IL-6, and CRP were higher in persons who died during the follow-up than in those who survived. When sex, education, cardiovascular disease, 
diabetes, cancer, history of infections, high density lipoprotein cholesterol, Mini-Mental State Examination, body mass index, smoking status, and exercise were adjusted for, only IL-1ra was a significant predictor of mortality. Persons in the upper tertiles of both CRP and IL-1ra, or in the upper tertile of all three markers, had higher mortality than those who were not in the upper tertile in any of the markers (Jylhä et al. 2007).

Furthermore, accumulated data strongly suggest that chronic upregulation of pro-inflammatory genes and molecules as pro-inflammatory cytokines, i.e. IL$1 \beta$, IL-6, Tumor necrosis Factor (TNF)- $\alpha$, and cyclooxygenase (COX)- 2 are induced during the ageing process. Moreover, the gene expression of these inflammatory markers is modulated by the redoxsensitive transcription factor, NF-kB, which is activated by the IKK/NIK and MAPK pathways. The activation of the network of NF-kB-dependent, proinflammatory molecules appears to be the molecular mechanism underlying numerous age-related diseases including dementia, cardiovascular disease, cancer, obesity, metabolic syndrome, and osteoporosis (Chung et al. 2009).

However, a wide range of different aetiological factors is likely to contribute to increased low-grade inflammatory activity in elderly populations including the contribution of different tissues (adipose tissue, muscle), organs (brain, liver), immune system and ecosystems (gut microbiota) (Cevenini et al. 2010). Furthermore, a recent hypothesis suggests that the reduction in lifetime exposure to infectious diseases and other sources of inflammation, the cohort mechanism, contributes to the historical decline in old-age mortality, suggesting long-life pathogen burden as the most important factor for age-related inflammation. Accordingly, some studies have linked an individual exposure to past infection to levels of chronic inflammation and to increased risk of heart attack, stroke, and cancer (Finch and Crimmins 2004; Licastro et al. 2005; Vasto et al. 2007).

On the other hand, persistent peripheral multibacteria infection such as periodontitis (PD), associated with gram-negative anaerobic bacteria capable of exhibiting localized and systemic infections in the host, has been proposed as a possible aggravating cofactor in subjects with vascular disease. In PD the balance between bacteria and host response is altered, resulting in a production of high levels of pro-inflammatory mediators and low levels of antiinflammatory ones. Locally, these molecules act in concert to amplify the inflammatory reaction and activate the effector mechanisms responsible for tissue destruction. Moreover, inflammatory chemokines and cytokines are elevated in plasma of PD patients, thus, contributing to the systemic inflammatory burden and exerting potential systemic effects. Data regarding the PD association with neurodegenerative disorders are lacking; however, it seems plausible that, during PD, cytokines increase in $\mathrm{AD}$ brain due to stimulation of trigeminal nerve fibres, or directly from bacterial products and/or bacteria. It seems relevant that in the NUN study a relationship between teeth loss and AD was found, but only in APO $\varepsilon 4$ negative subjects (Candore et al. 2009, 2010a, b).

Is "inflamm-ageing" compatible with longevity, i.e. 100 years of age or more? The answer is positive and apparently paradoxical, since, even if centenarians have an increased level of inflammatory mediators in comparison to old subjects and they are very frail, they also have high level of anti-inflammatory cytokines together with protective genotypes. Therefore, we think that the most proper approach to tackle and contain age-related inflammation, "inflammageing", is not only to identify the more suitable chemical therapies, but also to better identify the physiological molecules that are more likely present in long lived subjects and influence at the same time different organs of the body with pleiotropic characteristics. Indeed, since inflamm-ageing is a pleiotropic process where many different factors interact, in the same way pleiotropic interventions acting concomitantly on different targets and at different levels are to be preferred and chosen (Franceschi et al. 2007; Capri et al. 2008; Cevenini et al. 2010).

\section{Immunosenescence}

Inflamm-ageing is strictly linked to Immunosenescence, a complex process in which different immunological functions are impaired, others are remodelled (Candore et al. 2009; Vasto et al. 2010). Centenarians, a model of "successful-ageing", are a striking expression of this phenomenon (Franceschi et al. 1995). It is believed that immunosenescence is a major contributory factor to the increased frequency of morbidity and mortality among elderly. It renders 
elderly increasingly susceptible to infectious diseases, to the resurgence of latent infections, and also to infection by opportunistic organisms. In addition, there is a decline in the protective effect of vaccination in the elderly (Grubeck-Loebenstein et al. 2009).

Lifelong and chronic antigenic load results the major driving force of immunosenescence, which impacts on lifespan by reducing the number of virgin antigen-non experienced $\mathrm{T}$ cells, and filling the immunological space with expanded clones of memory and effector, antigen-experienced $\mathrm{T}$ cells. Thus, the repertoire of cells available to respond to antigenic challenge from previously unencountered pathogens is shrinking (Franceschi et al. 2000; De Martinis et al. 2005). Several studies have underlined the importance of ubiquitous viruses causing chronic latent infections, such as Herpes Viruses, in determining characteristic aspects of T-cell branch senescence such as the progressive exhaustion of naive lymphocytes, the increase in memory cells and the T repertoire shrinkage. Particularly, the Herpes virus Cytomegalovirus (CMV) seropositivity was associated with many of the same phenotypic and functional alterations of T-cell immunity considered as biomarkers in ageing. CMV is the prime driving force behind most of the oligoclonal expansions and altered phenotypes and functions of CD8 cells in the elderly. CMV-specific lymphocytes represent, even in immunocompetent subjects, a sizable proportion of both the $\mathrm{CD} 8+$ and the $\mathrm{CD} 4+$ memory compartment and they increase with age. Furthermore, the accumulation of these cells is due to the expansion of terminally differentiated exhausted lymphocytes and expanded clones restricted towards specific epitopes (Pawelec et al. 2004, 2005, 2009). In addition, very old subjects with a health status impaired by the most common age-related diseases exhibit an increase in CMV-specific effectors $\mathrm{T}$ cells, mostly $\mathrm{CD} 4+$, with a parallel increase in anti-CMV $\mathrm{IgG}$ antibodies (Trzonkowski et al. 2003; High 2005). The increase in cellular specific responses required to cope with CMV reactivation during ageing produces an increase in lymphocytes that release pro-inflammatory cytokines contributing to pro-inflammatory status involved in several age-related diseases (Trzonkowski et al. 2003; Vescovini et al. 2007; Sansoni et al. 2008; Candore et al. 2009; Cevenini et al. 2010).

Even though immune-gerontologists are fascinated by $\mathrm{T}$ cells, the B cell compartment is also affected in the elderly. In fact the number of B lymphocytes is decreased in aged humans, the B-cell repertoire is influenced by ageing during an immune response as described both in mice and in humans (Szabo et al. 2004); furthermore, it has recently been demonstrated that the decreased B cell diversity in old age is correlated with poor health status (Gibson et al. 2009). Moreover, even if the total serum immunoglobulin levels don't change, we have reported an age-related increase in IgG and IgA levels, and a decrease in IgM levels and $\operatorname{IgD}$ in elderly (Listì et al. 2006), so suggesting a shift from the naïve compartment of the $\mathrm{B}$ cell branch towards the memory compartment. However, the data are controversial as we and others (Gupta et al. 2005; Colonna-Romano et al. 2008) have demonstrated a significant decrease in naïve CD27$\mathrm{B}$ lymphocytes and a not significant reciprocal increase in CD27+ memory B cells, whereas others (Shi et al. 2005) have shown that CD27+ memory B cells, particularly IgD+IgM+CD27+ "IgM memory B cells", involved in the response to pneumococcal antigens, decrease dramatically in elderly. In fact, on the basis of the surface expression of CD27 and IgD B cells can be divided in (1) IgD+CD27- naïve B cells, (2) $\mathrm{IgD}+\mathrm{CD} 27+$ memory unswitched cells, and (3) IgD-CD27+ classic memory switched $(\mathrm{IgG}+$ or IgA+) cells (Shi et al. 2003).

In aged we have recently demonstrated that a double-negative (DN) IgD-CD27- B cell subset is significantly increased (Colonna-Romano et al. 2008, 2009). Moreover, this DN population although lacks CD27, has been described as a memory B cell population, and is expanded in patient with active lupus and healthy subjects exposed to respiratory sincitial virus (Wei et al. 2007; Sanz et al. 2008). In addition in a recent study (Colonna-Romano et al. 2010) we have analyzed B cell subsets in healthy centenarian offspring ( $\mathrm{CO}$ ) compared to age-matched controls to see whether any difference exists to explain the familiar increased lifespan expectancy of CO. In this population we didn't observe the typical näive/memory shift observed in elderly. In particular we didn't see the increase of DN (IgD-CD27-) B cells. This observation suggests that in $\mathrm{CO}$ the ability of bone marrow to generate $\mathrm{B}$ cells probably is not impaired as in elderly or, alternatively, that the systemic milieu might be different in these "genetically advantaged" people. This is an interesting hypothesis for two reasons. The first since it is well known that $\mathrm{CO}$ are a good model to study longevity 
(Colonna-Romano et al. 2010); the second one because it is known that memory and naive B cells produce different cytokines, as the latter produce the anti-inflammatory cytokine IL-10 and the former the pro-inflammatory cytokines TNF. Naive and memory B cells also express different toll receptors (TLRs) and so they have a regulatory role in the chronic infections against viruses and bacteria also producing cytokines and chemokines. This suggests that the immune-inflammation is also related to the B cell branch of the immune system in elderly people since they are able to produce different chemokines and cytokines (Chan et al. 1999; Harris et al. 2000; Martin and Chan 2006). Hence, B cell studies are relevant to our understanding of instructive immunity, inflammation, genetic background and longevity.

Age-related changes in lymphocytes have been well studied, whereas findings on the effect of ageing on phagocytic cell function and redox state are not consistent in the scientific literature, and there are few data on centenarians (Franceschi et al. 1995; Di Lorenzo et al. 1999; Miyaji et al. 2000; AlonsoFernández et al. 2008). On the other hand, several aspects of the innate immune response are affected by normal human ageing, resulting in a reduced ability to provide the immediate response to pathogens and also to integrate with and influence the acquired immune response. The mechanisms underlying these changes include alterations in the activity of a variety of innate immune cell receptors and their downstream signaling pathways, as well as changes to the numbers of certain cells within blood (Panda et al. 2009). This loss of innate cell function has consequences for immunity and longevity, with reduced neutrophil and NK cell activity predictive of increased mortality in old adults and dysregulation of TLR function affecting vaccine responsiveness and hyper-responsiveness to viral infection in aged individuals (Niwa et al. 1989; Di Lorenzo et al. 1999; Ogata et al. 2001; van Duin et al. 2007; Kong et al. 2008; Panda et al. 2009). In a recent report, several neutrophil functions (adherence, chemotaxis, phagocytosis, and stimulated and nonstimulated intracellular superoxide anion levels) and antioxidant parameters (glutathione levels and catalase activity) were measured in peripheral blood neutrophil suspension in centenarians, young adults, and middle-aged adults. Neutrophil functions of the middle-aged group were worse than those of young adults and centenarians (lower chemotaxis and phagocytosis and higher adherence and superoxide anion levels). The neutrophil functions of the centenarians were closer to those of the young adults. This study found well-preserved antioxidant status in peripheral blood neutrophils, which are responsible for initiating the inflammatory cascade. When inflammatory processes escape these antioxidant regulatory mechanisms, systemic effects may be provoked (such as increasing inflammatory cytokines and other oxidative stress molecules), and these could contribute to many chronic age-associated diseases and to higher mortality. The centenarians studied had well-preserved antioxidant ability, low superoxide anion production, and nonaltered peripheral blood neutrophil functions. Therefore, these characteristics could be goals that would enable them to avoid ageassociated diseases and attain longevity. It is possible that the redox state of neutrophils provides centenarians with better health and prevents age-related diseases (De la Fuente et al. 2005; Viveros et al. 2007; Alonso-Fernández et al. 2008).

\section{Genetic background}

An impressive and coherent series of epidemiological data in different populations indicate the presence of a strong familial component of longevity. These studies demonstrate that parents, siblings and offspring of long-lived subjects (but not the spouses of the longlived subjects who shared with them most part of their adult life) have a significant survival advantage, a higher probability to have been or to became longliving persons and to have a lower risk to undergo the most important age-related diseases, such as cardioand cerebro-vascular diseases, diabetes and cancer, when compared to the appropriate control. The genetics of longevity appears to be quite peculiar, owing to the fact that it regards the post-reproductive period of life, a period largely non predicted by evolution and characterized by a progressive decrease of the force of selection. Thus, centenarians may have originated from an initial frail part of the cohort which was able to survive at younger (reproductive) age and it was later allowed to exploit genes useful in the postreproductive period of life. Vice versa it can be hypothesized that genes neutral or dangerous at younger age can became useful at old or extremely old age (Capri et al. 2008; Franceschi et al. 2008). 
The majority of above described inflammatory aspects, that characterize ageing, are also detectable in extreme longevity, where a higher frequency of genetic markers associated with a reduced proinflammatory ability seems to counteract the onset of the main age-related inflammatory disorders. The data on the determinants of human longevity indicate that centenarians are a good choice for the study of longevity, because they represent an extreme phenotype, i.e., the survival tail of the population who escaped neonatal mortality, pre-antibiotic era illnesses, and fatal outcomes of age-related complex diseases. Centenarians are quite capable of mounting effective inflammatory responses; however, inflammatory status, correlated to increasing risk of developing frailty and diseases, is compensated by the concomitant development of strong and effective antiinflammatory responses. Centenarians are remarkably enriched in "good" genotypes and show opposite frequencies of "bad" genotypes in comparison with patients affected by major age-related pathologies, such as CVD, AD and cancer (Candore et al. 2006, 2007; Franceschi et al. 2007; Vasto et al. 2008, 2009).

In fact, in the last years investigations aimed to identify a possible genetic propensity to produce high or low levels of pro-inflammatory cytokines and other pro-inflammatory molecules were started. Different teams of investigators were involved in this ambitious task. This critical mass allowed to increase the number of inflammatory cytokine genes found associated to longevity (IL-6, TNF- $\alpha$ ) as well as those coding for anti-inflammatory cytokines such as IL-10 and tumor growth factor- $\beta 1$. In addition genes coding for molecules crucial for the recognition of pathogens and the start of immune response such as TLR4 and other which plays an important role in inflammatory responses (pyrin, connexin, $\alpha 1$-antitrypsin, 5-lipoxygenase, COX-2, chemokine receptor-5) have been also shown to be associated to longevity (Candore et al. 2006; Salvioli et al. 2006; Balistreri et al. 2008; Listì et al. 2008; Ostan et al. 2008; Rea et al. 2006; Di Bona et al. 2009; Vasto et al. 2009). On the whole, these data suggest that a pro-inflammatory genotype is unfavourable to reach extreme longevity in good health and likely favours the onset of age-related diseases such as CVD and AD which are the leading causes of mortality and disability in the elderly. Within this perspective, healthy ageing and longevity are likely the result not only of a lower propensity to mount inflammatory responses but also of an efficient anti-inflammatory network, which in unsuccessful ageing fail to fully neutralize the inflammatory processes consequent to the lifelong antigenic burden and exposure to damaging agents. Such a global imbalance can be a major driving force for frailty and common age-related pathologies, and should be addressed and studied within an evolutionary-based systems biology perspective. Moreover, many associations between gene variants and longevity have been found only in Italian population. This should not be unexpected, since ageing and longevity are complex traits resulting not only and not exclusively from genetics, but rather from the interactions between genetics, environment and chance (Capri et al. 2008; Franceschi et al. 2008).

A typical example is represented by IL- $6-174$ single nucleotide polymorphism (SNP). Several studies have assessed changes in frequency of -174 IL-6 SNP with age. If IL-6 tracks with disability and agerelated diseases (Vasto et al. 2010), then there should be reduction, in the oldest old, of the frequency of homozygous GG subjects, who produce higher IL-6 levels. However, discordant results have been obtained. To explore the relationship between this SNP and longevity, we analyzed individual data on long-living subjects and controls from eight casecontrol studies conducted in Europeans, using metaanalysis. There was no significant difference in the IL6 genotype between the oldest old and controls, but there was significant between-study heterogeneity. In a subgroup analyses when male centenarians from the three Italian studies were included, the frequency of the IL-6 -174 GG genotype was significantly lower than the other genotypes, with no evidence of heterogeneity. Our data supports a negative association between the GG genotype of IL-6 SNP and longevity in Italian centenarians, with males who carry the genotype being two times less likely to reach extreme old age compared with subjects carrying CC or CG genotypes. These findings were not replicated in other European groups suggesting a possible interaction between genetics, sex and environment in reaching longevity (Di Bona et al. 2009).

In this perspective, it is conceivable that longevity can be achieved by different combinations of these three components, and thus, when gene pool and environment change, the combinations leading to longevity change as well. However, these data 
prompt consideration of the role that antagonistic pleiotropy plays in diseases and in longevity. Our immune system has evolved to control pathogens, so pro-inflammatory responses are likely to be evolutionarily programmed to resist fatal infections. Yet genetic backgrounds promoting pro-inflammatory responses play an opposite role in unsuccessful ageing and in longevity, such that CVD and AD are a late consequence of evolutionary programming for a pro-inflammatory response to resist infections at an early age. Genetic polymorphisms responsible for a low inflammatory response may better control inflammatory responses involved in these diseases. So, these polymorphisms might result in an increased chance of long life-span in an environment with reduced pathogen load (Caruso et al. 2005).

Acknowledgement This work was supported by grants from Palermo University to GC, CC and GCR.

\section{References}

Alonso-Fernández P, Puerto M, Maté I, Ribera JM, de la Fuente M (2008) Neutrophils of centenarians show function levels similar to those of young adults. J Am Geriatr Soc 56:2244-2251

Balistreri CR, Candore G, Caruso M, Incalcaterra E, Franceschi C, Caruso C (2008) Role of polymorphisms of CCchemokine receptor-5 gene in acute myocardial infarction and biological implications for longevity. Haematologica 93:637-638

Candore G, Balistreri CR, Grimaldi MP, Listì F, Vasto S, Caruso $\mathrm{M}$ et al (2006) Opposite role of pro-inflammatory alleles in acute myocardial infarction and longevity: results of studies performed in a Sicilian population. Ann N Y Acad Sci 1067:270-275

Candore G, Balistreri CR, Caruso M, Grimaldi MP, Incalcaterra E, Listì F et al (2007) Pharmacogenomics: a tool to prevent and cure coronary heart disease. Curr Pharm Des 13:3726-3734

Candore G, Balistreri CR, Bulati M, Colonna-Romano G, Di Bona D, Forte GI et al (2009) Immune-inflammatory responses in successful and unsuccessful ageing. G Gerontol 57:145-152

Candore G, Caruso C, Jirillo E, Magrone T, Vasto S (2010a) Low grade inflammation as a common pathogenetic denominator in age-related diseases: novel drug targets for anti-ageing strategies and successful ageing achievement. Current Pharm Des 16:584-596

Candore G, Bulati M, Caruso C, Castiglia L, Colonna-Romano G, Di Bona D et al (2010b) Inflammation, cytokines, immune response, apolipoprotein E, cholesterol, and oxidative stress in Alzheimer disease: therapeutic implications. Rejuvenation Res 13:301-313
Capri M, Salvioli S, Monti D, Caruso C, Candore G, Vasto S et al (2008) Human longevity within an evolutionary perspective: the peculiar paradigm of a post-reproductive genetics. Exp Gerontol 43:53-60

Caruso C, Candore G, Colonna-Romano G, Lio D, Franceschi C (2005) Inflammation and life-span. Science 307:208-209

Cevenini E, Caruso C, Candore G, Capri M, Nuzzo D, Duro G et al (2010) Age-related inflammation: the contribution of different organs, tissues and systems. How to face it for therapeutic approaches. Current Pharm Des 16:609-618

Chan OT, Madaio MP, Shlomchik MJ (1999) The central and multiple roles of B cells in lupus pathogenesis. Immunol Rev 169:107-121

Chung HY, Cesari M, Anton S, Marzetti E, Giovannini S, Seo AY et al (2009) Molecular inflammation: underpinnings of aging and age-related diseases. Ageing Res Rev 8:18-30

Colonna-Romano G, Bulati M, Aquino A, Vitello S, Lio D, Candore $G$ et al (2008) B cell immunosenescence in elderly and centenarians. Rejuvenation Res 11:433-439

Colonna-Romano G, Bulati M, Aquino A, Pellicanò M, Vitello S, Lio D et al (2009) A double-negative (IgD-CD27-) B cell population is increased in the peripheral blood of elderly people. Mech Ageing Dev 130:681-690

Colonna-Romano G, Buffa S, Bulati M, Candore G, Vaso S, Lio D et al (2010) B cells compartment in centenarian offspring and old people. Current Pharm Des 16:604-608

De la Fuente M, Hernanz A, Vallejo MC (2005) The immune system in the oxidative stress conditions of aging and hypertension: favorable effects of antioxidants and physical exercise. Antioxid Redox Signal 7:1356-1366

De Martinis M, Franceschi C, Monti D, Ginaldi L (2005) Inflamm-ageing and lifelong antigenic load as major determinants of ageing rate and longevity. FEBS Lett 579:2035-2039

Di Bona D, Vasto S, Capurso C, Christiansen L, Deiana L, Franceschi C et al (2009) Effect of interleukin-6 polymorphisms on human longevity: a systematic review and meta-analysis. Ageing Res Rev 8:36-42

Di Lorenzo G, Balistreri CR, Candore G, Cigna D, Colombo A, Romano GC et al (1999) Granulocyte and natural killer activity in the elderly. Mech Ageing Dev 108:25-38

Finch CE, Crimmins EM (2004) Inflammatory exposure and historical changes in human life-spans. Science 305:1736-1739

Franceschi C, Monti D, Sansoni P, Cossarizza A (1995) The immunology of exceptional individuals: the lesson of centenarians. Immunol Today 16:12-16

Franceschi C, Bonafe M, Valensin S (2000) Human immunosenescence: the prevailing of innate immunity, the failing of clonotypic immunity, and the filling of immunological space. Vaccine 18:1717-1720

Franceschi C, Capri M, Monti D, Giunta S, Olivieri F, Sevini F et al (2007) Inflammaging and anti-inflammaging: a systemic perspective on aging and longevità emerged from studies in humans. Mech Ageing Dev 128:92-105

Franceschi C, Motta L, Motta M, Malaguarnera M, Capri M, Vasto $S$ et al (2008) The extreme longevity: the state of the art in Italy. Exp Gerontol 43:45-52

Gibson KL, Wu YC, Barnett Y, Duggan O, Vaughan R, Kondeatis E et al (2009) B-cell diversity decreases in old 
age and is correlated with poor health status. Aging Cell $8: 18-25$

Goto M (2008) Inflammaging (inflammation + aging): a driving force for human aging based on an evolutionarily antagonistic pleiotropy theory? Biosci Trends 2:218-230

Grubeck-Loebenstein B, Della Bella S, Iorio AM, Michel JP, Pawelec G, Solana R (2009) Immunosenescence and vaccine failure in the elderly. Aging Clin Exp Res 21:201-209

Gupta S, Su H, Bi R, Agrawal S, Gollapudi S (2005) Life and death of lymphocytes: a role in immunosenescence. Immun Ageing 2:2

Harris DP, Haynes L, Sayles PC, Duso DK, Eaton SM, Lepak $\mathrm{NM}$ et al (2000) Reciprocal regulation of polarized cytokine production by effector $\mathrm{B}$ and $\mathrm{T}$ cells. Nat Immunol 1:475-482

High KP (2005) Chronic infection and frailty: surrogate markers, associations, and causality. J Am Geriatr Soc 53:906-908

Jylhä M, Paavilainen P, Lehtimäki T, Goebeler S, Karhunen PJ, Hervonen A et al (2007) Interleukin-1 receptor antagonist, interleukin-6, and C-reactive protein as predictors of mortality in nonagenarians: the vitality $90+$ study. J Gerontol A Biol Sci Med Sci 62:1016-1021

Kong KF, Delroux K, Wang X, Qian F, Arjona A, Malawista SE et al (2008) Dysregulation of TLR3 impairs the innate immune response to West Nile virus in the elderly. J Virol 82:7613-7623

Licastro F, Candore G, Lio D, Porcellini E, Colonna-Romano $\mathrm{G}$, Franceschi $\mathrm{C}$ et al (2005) Innate immunity and inflammation in ageing: a key for understanding age related diseases. Immun Ageing 2:8

Listì F, Candore G, Modica MA, Russo M, Di Lorenzo G, Esposito-Pellitteri M et al (2006) A study of serum immunoglobulin levels in elderly persons that provides new insights into B cell immunosenescence. Ann N Y Acad Sci 1089:487-495

Listì F, Caruso M, Incalcaterra E, Hoffmann E, Caimi G, Balistreri CR et al (2008) Pro-inflammatory gene variants in myocardial infarction and longevity: implications for pharmacogenomics. Curr Pharm Des 14:2678-2685

Martin F, Chan AC (2006) B cell immunobiology in disease: evolving concepts from the clinic. Annu Rev Immunol 24:467-496

Miyaji C, Watanabe H, Toma H, Akisaka M, Tomiyama K, Sato Y et al (2000) Functional alteration of granulocytes, NK cells, and natural killer T cells in centenarians. Hum Immunol 61:908-916

Niwa Y, Kasama T, Miyachi Y, Kanoh T (1989) Neutrophil chemotaxis, phagocytosis and parameters of reactive oxygen species in human aging: cross-sectional and longitudinal studies. Life Sci 44:1655-1664

Oeppen J, Vaupel JW (2002) Demography. Broken limits to life expectancy. Science 296:1029-1031

Ogata K, An E, Shioi Y, Nakamura K, Luo S, Yokose N et al (2001) Association between natural killer cell activity and infection in immunologically normal elderly people. Clin Exp Immunol 124:392-397

Olshansky SJ, Ault AB (1986) The fourth stage of the epidemiologic transition: the age of delayed degenerative diseases. Milbank Q 64:355-391
Ostan R, Bucci L, Capri M, Salvioli S, Scurti M, Pini E et al (2008) Immunosenescence and immunogenetics of human longevity. Neuroimmunomodulation 15:224-240

Panda A, Arjona A, Sapey E, Bai F, Fikrig E, Montgomery RR et al (2009) Human innate immunosenescence: causes and consequences for immunity in old age. Trends Immunol 30:325-333

Pawelec G, Akbar A, Caruso C, Effros R, Grubeck-Loebenstein B, Wikby A (2004) Is immunosenescence infectious? Trends Immunol 25:406-410

Pawelec G, Akbar A, Caruso C, Solana R, Grubeck-Loebenstein B, Wikby A (2005) Human immunosenescence: is it infectious? Immunol Rev 205:257-268

Pawelec G, Derhovanessian E, Larbi A, Strindhall J, Wikby A (2009) Cytomegalovirus and human immunosenescence. Rev Med Virol 19:47-56

Rattan SI (2008) Hormesis in aging. Ageing Res Rev 7:63-78

Rea IM, Candore G, Cavallone L, Olivieri F, Cardelli M, Franceschi C et al (2006) Longevity. In: Vandenbroeck K (ed) Cytokine gene polymorphisms in multifactorial conditions. CRC Press, Boca Raton, pp 379-394

Salvioli S, Olivieri F, Marchegiani F, Cardelli M, Santoro A, Bellavista E et al (2006) Genes, ageing and longevity in humans: problems, advantages and perspectives. Free Radic Res 40:1303-1323

Sansoni P, Vescovini R, Fagnoni F, Biasini C, Zanni F, Zanlari L et al (2008) The immune system in extreme longevity. Exp Gerontol 63:61-65

Sanz I, Wei C, Lee FE, Anolik J (2008) Phenotypic and functional heterogeneity of human memory B cells. Semin Immunol 20:67-82

Shi Y, Agematsu K, Ochs HD, Sugane K (2003) Functional analysis of human memory B-cell subpopulations: $\mathrm{IgD}+\mathrm{CD} 27+\mathrm{B}$ cells are crucial in secondary immune response by producing high affinity IgM. Clin Immunol 108:128-137

Shi Y, Yamazaki T, Okubo Y, Uehara Y, Sugane K, Agematsu K (2005) Regulation of aged humoral immune defense against pneumococcal bacteria by IgM memory B cell. J Immunol 175:3262-3267

Szabo P, Li F, Mathew J, Lillvis J, Weksler ME (2004) Evolution of B-cell clonal expansions with age. Cell Immunol 231:158-167

Troen BR (2003) The biology of aging. Mt Sinai J Med 70:322

Trzonkowski P, Myśliwska J, Szmit E, Wieckiewicz J, Lukaszuk K, Brydak LB et al (2003) Association between cytomegalovirus infection, enhanced proinflammatory response and low level of anti-hemagglutinins during the anti-influenza vaccination-an impact of immunosenescence. Vaccine 21:3826-3836

van Duin D, Allore HG, Mohanty S, Ginter S, Newman FK et al (2007) Prevaccine determination of the expression of costimulatory B7 molecules in activated monocytes predicts influenza vaccine responses in young and older adults. J Infect Dis 195:1590-1597

Vasto S, Candore G, Balistreri CR, Caruso M, ColonnaRomano G, Grimaldi MP et al (2007) Inflammatory networks in ageing, age-related diseases and longevity. Mech Ageing Dev 128:83-91 
Vasto S, Candore G, Listì F, Balistreri CR, Colonna-Romano $\mathrm{G}$, Malavolta $\mathrm{M}$ et al (2008) Inflammation, genes and zinc in Alzheimer's disease. Brain Res Rev 58:96-105

Vasto S, Carruba G, Lio D, Colonna-Romano G, Di Bona D, Candore $\mathrm{G}$ et al (2009) Inflammation, ageing and cancer. Mech Ageing Dev 130:40-45

Vasto S, Scapagnini G, Bulati M, Candore G, Castiglia L, Colonna-Romano $\mathrm{G}$ et al (2010) Biomarkers of ageing. Front Biosci 2:392-402

Vescovini R, Biasini C, Fagnoni FF, Telera AR, Zanlari L, Pedrazzoni $\mathrm{M}$ et al (2007) Massive load of functional effector CD4+ and CD8+ T cells against cytomegalovirus in very old subjects. J Immunol 179:4283-4291

Viveros MP, Arranz L, Hernanz A, Miquel J, De la Fuente M (2007) A model of premature aging in mice based on altered stress-related behavioral response and immunosenescence. Neuroimmunomodulation 14:157-162

Wei C, Anolik J, Cappione A, Zheng B, Pugh-Bernard A, Brooks J et al (2007) A new population of cells lacking expression of CD27 represents a notable component of the $\mathrm{B}$ cell memory compartment in systemic lupus erythematosus. J Immunol 178:6624-6633 